\title{
10 Volt Automated Direct Comparison of Two Cryocooled Programmable Josephson Voltage Standards
}

\author{
Alain Rüfenacht ${ }^{1}$, Yi-hua Tang ${ }^{2}$, Anna E. Fox ${ }^{1}$, Paul D. Dresselhaus ${ }^{1}$, Charles J. Burroughs ${ }^{1}$, \\ Robert E. Schwall ${ }^{1}$, and Samuel P. Benz ${ }^{1}$ \\ ${ }^{1}$ National Institute of Standards and Technology NIST, Boulder, CO 80305, USA \\ ${ }^{2}$ National Institute of Standards and Technology NIST, Gaithersburg, MD 20899, USA \\ Email: alain.rufenacht@nist.gov
}

\begin{abstract}
We have performed direct de comparisons between two cryocooled $10 \mathrm{~V}$ programmable Josephson voltage standards utilizing an automated synchronization scheme for the voltage reversals, which enables the use of a high sensitivity null detector on its $3 \mu \mathrm{V}$ range by preventing any overload condition. No switches or manual operations are necessary to protect the null detector. Comparing the two systems under various test conditions provides robust verification of ideal system performance, and enables verification of the key components of the uncertainty budget for both the measurement methods and system operations.
\end{abstract}

Index Terms - Josephson arrays, Precision measurements, Uncertainty, Voltage measurement.

\section{INTRODUCTION}

The NIST programmable Josephson voltage standard (PJVS) with an output voltage of $10 \mathrm{~V}$ has been fully implemented on a cryocooler [1]. Turn-key cryocooled PJVS systems are replacing conventional Josephson voltage standards, not only in national metrology institutes (NMI), but also in primary calibration laboratories (PCL). Direct PJVS to PJVS comparisons [2] are not only becoming prevalent for NMI key comparisons, but are also essential to qualify the performance of PJVS systems disseminated to the PCL. The lowest Type-A uncertainty in direct voltage standard comparisons is generally achieved with the use of an analog null detector [2,3]. However, the use of an analog detector often demands fastidious manual operations to prevent any overloading during the PJVS polarity reversals required to eliminate the contributions of thermal electromotive forces (EMFs). If the input stage of the analog nanovoltmeter is overloaded, it may take several minutes to completely recover.

This paper presents the first fully automated direct comparison of two cryocooled $10 \mathrm{~V}$ PJVS systems. We have developed a synchronization method to simultaneously reverse the polarity of both PJVS systems, which prevents overloading the analog detector, while eliminating the need for a manual low thermal EMF switch to protect the detector input. The main advantage of this method is the ability to automatically acquire longer data sets, thus improving the statistical uncertainty of the comparison and eliminating the risk of improper manual operation that may produce an accidental overload.

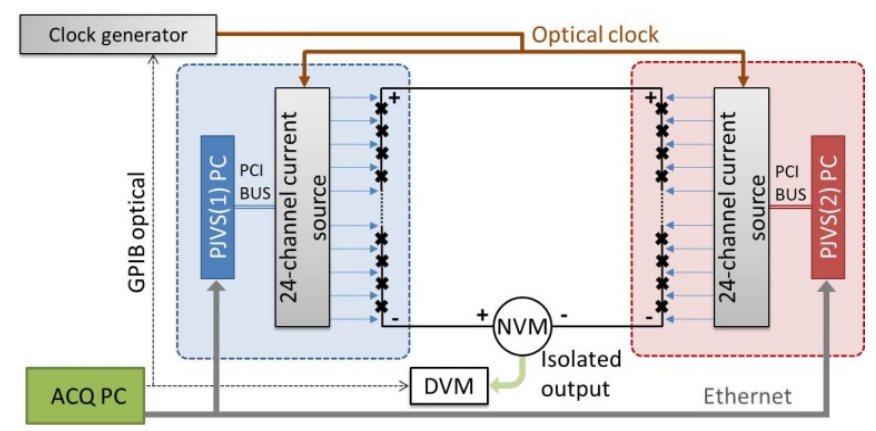

Fig. 1. Block diagram of the automated comparison between the two PJVS systems measured with an analog nanovoltmeter (NVM).

\section{MEASUREMENT SETUP AND SYNCHRONIZATION}

The measurement setup is presented in Fig. 1. An analog nanovoltmeter (NVM), EM N11 ${ }^{1}$, was used as a null detector to measure the voltage difference between the two cryocooled voltage standards, PJVS(1) and PJVS(2). The isolated output of the NVM was connected to a digital voltmeter (DVM) for digital readout. The acquisition computer (ACQ PC) controls the amplitude of both PJVS systems by means of an Ethernet connection. The two 24-channel current sources for each PJVS are isolated from ground, and the two microwave generators (not shown) are locked to the same $10 \mathrm{MHz}$ frequency reference (derived from the NIST atomic clock). An arbitrary waveform generator provides two optical clock signals for synchronization, one for each PJVS current bias source. The two PJVS systems are identical, with comparable cable lengths (optical fiber for the clock input, current bias leads, voltage output leads).

The magnitude of the voltage at the input of the NVM, must always remain within the range selected to prevent an overload. Each synchronous change of voltage uses a fourlevel waveform ${ }^{2}$ (Table 1) triggered by four successive clock pulses at $5 \mathrm{kHz}$ (Fig. 2). The waveform is first loaded in the memory of both current sources and is programmed to run for

\footnotetext{
1 Certain commercial equipment, instruments, or materials are identified in this report to facilitate understanding. Such identification does not imply recommendation or endorsement by NIST, nor does it imply that the materials or equipment that are identified are necessarily the best available for the purpose.

${ }^{2}$ Minimum waveform size allowed by the current source.
} 
a single cycle. Once the waveform is completed, the voltage of each PJVS remains at the final voltage level selected.

Table 1 . Waveform used to synchronously switch the voltage output polarity of both arrays

\begin{tabular}{|c|c|c|c|c|c|}
\hline \multirow{2}{*}{$\begin{array}{c}\text { Switching } \\
\text { mode }\end{array}$} & \multirow{2}{*}{$\begin{array}{c}\text { Start } \\
\text { voltage }\end{array}$} & \multicolumn{4}{|c|}{ Waveform (4 levels) } \\
\hline & & $\# 1$ & $\# 2$ & $\# 3$ & $\# 4$ (end) \\
\hline A (start) & $0 \mathrm{~V}$ & $0 \mathrm{~V}$ & $0 \mathrm{~V}$ & $10 \mathrm{~V}$ & $10 \mathrm{~V}$ \\
\hline $\mathbf{B}(+\rightarrow-)$ & $10 \mathrm{~V}$ & $0 \mathrm{~V}$ & $0 \mathrm{~V}$ & $-10 \mathrm{~V}$ & $-10 \mathrm{~V}$ \\
\hline $\mathbf{C}(-\rightarrow+)$ & $-10 \mathrm{~V}$ & $0 \mathrm{~V}$ & $0 \mathrm{~V}$ & $10 \mathrm{~V}$ & $10 \mathrm{~V}$ \\
\hline $\mathbf{D}$ (end) & $10 \mathrm{~V}$ & $0 \mathrm{~V}$ & $0 \mathrm{~V}$ & $0 \mathrm{~V}$ & $0 \mathrm{~V}$ \\
\hline
\end{tabular}

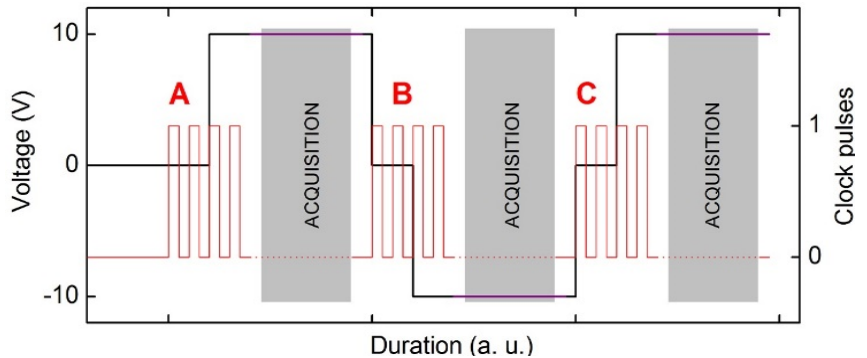

Fig. 2. Schematic of the waveform used to perform the synchronous switching between voltages, (A: start, B: from $+10 \mathrm{~V}$ to $-10 \mathrm{~V}$, and $\mathrm{C}$ : from $-10 \mathrm{~V}$ to $10 \mathrm{~V})$. The four clock pulses are shown in red. The data acquisition occurs once both PJVS output voltages are fully settled (gray area).
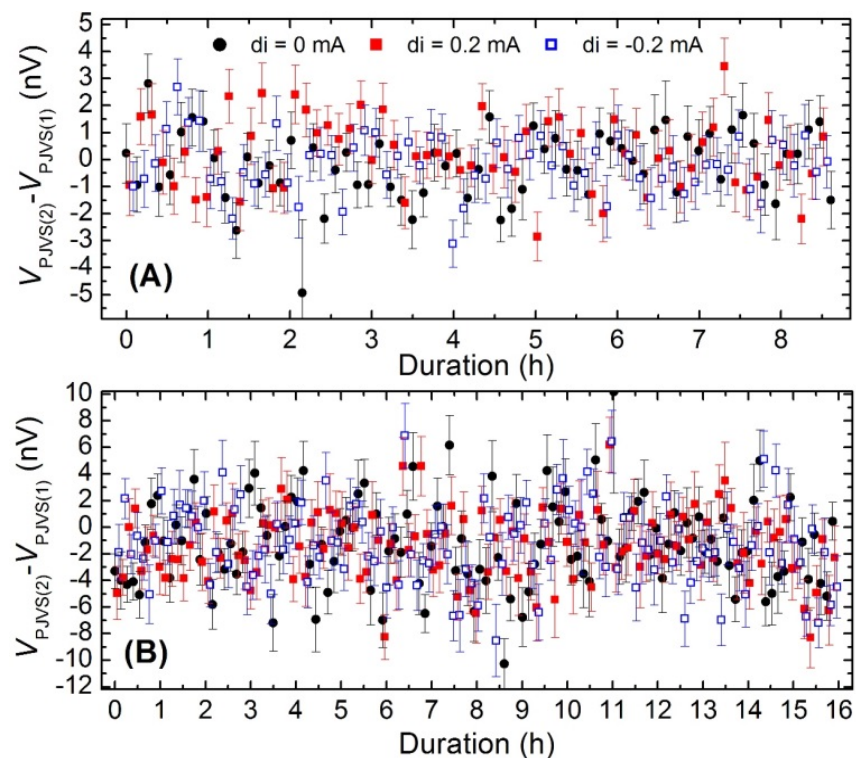

Fig. 3. Comparison results with (A) both systems floating from ground, and (B) with the low input of the NVM connected to earth ground.

\section{RESULTS}

Figure 3 shows the results of two multi-hour automated comparisons at $10 \mathrm{~V}$. Each value reported is calculated with a linear fit based on four polarity reversal sets "+-+-_" in order to remove the contributions of thermal EMFs. A polarity set consists of 15 DVM readings at 10 power line cycles. The
Type-A uncertainty shown (error bar, $k=1$ ) is extracted from the fit residuals [4]. No overload is observed during the polarity reversals with the NVM set to the $3 \mu \mathrm{V}$ range and the filter set to the maximal bandwidth (position 1), which confirms that the synchronization method is successful. To minimize potential error due to dielectric absorption time, the acquisition of each set starts $40 \mathrm{~s}$ after switching the voltage polarity. The data in both plots of Fig. 3 utilize the applied dither current technique [2], which confirms that both PJVS systems maintain a sufficient current margin when connected in series to the NVM throughout the full measurement time. The reported data are not corrected for the gain error of the NVM, since the measured relative correction is less than $5 \mathrm{pV} / \mathrm{nV}$ and is well below the noise floor of the measurement.

The result extracted from Fig. 3A shows excellent agreement between the two PJVS systems of $V_{\mathrm{PJVS}(2)}$ $V_{\mathrm{PJVS}(1)}=(-0.05 \pm 1.22) \mathrm{nV}$, where the uncertainty is the standard deviation $(k=1)$. Additional analysis of the $1 / f$ noise of the detector will be required to determine the standard deviation of the mean. However, when the low input of the NVM is connected to ground (Fig. 3B), the final result is $V_{\mathrm{PJVS}(2)}-V_{\mathrm{PJVS}(1)}=(-1.26 \pm 3.11) \mathrm{nV}$. Grounding the circuit modifies the leakage current distribution, which affects the result of the comparison and also slightly increases the noise of the measurement. Due to the complex distribution of the leakage current in PJVS systems, additional measurements are required to understand and evaluate the uncertainty contributions related to leakage.

\section{CONCLUSION}

We have performed automated comparisons between two cryocooled PJVS systems at $10 \mathrm{~V}$ dc. Synchronization of the two systems when switching between output levels enables the use of a high sensitivity null detector with a $\pm 3 \mu \mathrm{V}$ input range. No switches or manual operator tasks are required in these measurements, which minimizes wiring connections. In the final paper on this work, we will present a detailed uncertainty budget including further investigation of potential Type-B contributions.

\section{REFERENCES}

[1] A. Rüfenacht, et al., "Cryocooled $10 \mathrm{~V}$ Programmable Josephson Voltage Standard," IEEE Trans. Instrum. Meas., vol. 64, no. 6, pp. 1477-1482, Jun. 2015.

[2] S. Solve, et al., "Direct comparison of two NIST PJVS systems at $10 \mathrm{~V}, "$ Metrologia, vol. 50, no. 5, pp. 441-451, 2013.

[3] S. Djordjevic, et al., "Direct comparison between a programmable and a conventional Josephson voltage standard at the level of 10V," Metrologia, vol. 45, pp. 429-35, 2008.

[4] C. A. Hamilton, et al., "A compact transportable Josephson voltage standard," IEEE Trans. Instrum. Meas., vol. 46, no. 2, pp. 237-241, Apr. 1997. 